\title{
Exploration and Practice of Guidance Mode about Engineering Course's Graduation Project -----Guidance about Graduation Project for Students with Learning Difficulties
}

\author{
Bing Yan \\ School of Computer \& Computing Science, Zhejiang University City College, Hangzhou, Zhejiang, China (yanb@zucc.edu.cn)
}

\begin{abstract}
This text introduces how to guide engineering course's students with learning difficulties to implement graduation project. At first the text analyses some common methods to instruct graduation project in Colleges and Universities, and expounds the necessity to constitute a set of methods of the guidance and management about graduation project for the students with learning difficulties. Then it expounds the reforms of the guidance's and management's method about graduation project to take the students with learning difficulties to major in computer science for example, including the reform of the management mode about graduation project, the reform of the guidance process about graduation project, and the design of the content about graduation project, etc. Finally, the text shows the good effects on students with learning difficulties through a series of reforms.
\end{abstract}

Keywords_-graduation project, students with learning difficulties, teaching mode

\section{工科毕业设计指导方式改革的探索与实践 ----学习困难生的毕业设计指导}

\author{
严冰 \\ 浙江大学城市学院计算机与计算科学分院, 杭州, 浙江, 中国
}

摘 要 本文介绍了在工科毕业设计指导中如何针对学习困难生进行毕业设计指导与管理的改革探索。文章首先简要分析了现行 高校普遍采用的毕业设计指导方法, 并阐述了制定一套专门针对学习困难生的毕业设计指导与管理方法的必要性, 然后详细介绍了笔 者所在学院针对计算机专业的学习困难生开展的毕业设计指导与管理方法的改革, 具体包括毕业设计管理模式的改革、毕业设计指导 过程的改革以及毕业设计内容的设计等。最后说明了通过这一系列改革对学习困难生产生的积极效果。

关键词 毕业设计, 学习困难生, 教学模式

\section{1. 背景}

毕业设计是高校本科生教学环节中最后也是最重要的 一个环节。学生在该环节中要完成资料检索、外文资料翻 译、文献综述、开题报告、方案设计、方案实现（实验）、 论文撰写、论文答辩等工作。所以, 毕业设计是对本科生 运用所学的专业知识进行系统设计的能力的综合性总结和 检验, 是本科生进入工作岗位或再深造前的一次重要的实 践尝试。通过毕业设计, 将促进学生学会如何将理论知识 贯彻到实际应用中去, 为他们今后的就业或深造打下良好
的基础[1]。

现在高校普遍通行的毕业设计指导方法是一名教师指 导多名学生, 给每一位学生一个研究项目, 以学生为主开 展研究, 教师作一定的指导。这种模式对于前三年课程知 识掌握得不错的学生来说, 是能够顺利完成并达到预期效 果的, 但每个学校总有那么一些学习困难生, 他们前面三 年的课程虽然勉强及格了, 但却没有很好掌握, 最后一年 要让他们独立做理论研究或独立开发一个项目是有相当大 难度的。因此就往往导致这些学生一开始就知难而退选择 
了放弃, 浑浑哥哥度过最后一年, 最终或者不能通过毕业 论文答辩而毕不了业, 或者虽然碰运气通过论文答辩了却 其实根本不具备相应的专业知识, 白白浪费了一年。由于 近几年中国高校大规模扩招, 学生整体素质有所下降, 这 部分学生的数量还不少。有人因此提出取消大学生毕业设 计(论文)的观点。但如果因某些现实困难, 就简单地对毕业 设计(论文) “一除了之”, 无异于削足适履, 会更加降低学 生的综合能力。而且, 中国教育部在《关于加强普通高等 学校毕业设计(论文)工作的通知》中明确强调毕业设计的重 要性, 指出不仅不能取消毕业设计(论文), 还应进一步加强, 将其作为高校本科教学评估的一个关键指标[2]。

所以为了使学习困难生能顺利完成毕业设计, 在最后 一个教学环节中能真正达到强化专业知识、增长谋生本领 的目的, 制定一套专门针对学习困难生的毕业设计指导与 管理方法就显得很有必要。在过去的几届学生中, 我们着 手进行了学习困难生的毕业设计指导方式的改革, 并已经 取得了较好的效果。下面以计算机专业的毕业设计指导为 例, 具体介绍我们是如何针对学习困难生进行毕业设计指 导与管理的。

\section{2. 具体措施}

\section{1 毕业设计管理模式的改革[3]}

要使得针对学习困难生的这项毕业设计改革能顺利进 行, 组织管理工作尤为重要。只有建立了良好的一套毕业 设计管理机制, 才能保障各项工作能顺利开展, 才能切实 帮助学习困难生顺利完成毕业设计这个教学环节的任务。

因此, 我们首先成立了学习困难生毕业设计管理工作 小组, 管理工作小组由教学经验丰富、工作认真负责的若 干名教师组成, 管理工作小组的任务是全权负责学习困难 生的整个毕业设计过程的指导管理工作。

其次是挑选、组织学习困难生成为一个毕业设计辅导 班, 进行统一集中管理。我们选择学业成绩排名在年级后 四分之一的学生作为可以加入学习困难生毕业设计辅导班 的条件, 先向这些学生详细介绍辅导班的学生将如何开展 毕业设计, 再由学生自愿选择是否报名参加辅导班。最终 确定参加辅导班的人数一般是占全年级学生总数的 $15 \%$ 至 $20 \%$ 。

管理工作小组为学习困难生量身定制了一套毕业设计 指导与管理的方法。主要的思路是分阶段对辅导班的学生 进行集中培训 (具体内容见下节), 同时通过教师与学生的 双向选择, 管理工作小组的每个指导教师负者具体指导若 干名学生。也即管理模式的核心是采取集中培训与个别指 导相结合的原则。

\section{2 毕业设计指导过程的改革}

在为期一年的毕业设计期间, 首先对毕业设计辅导班 的学生集中进行几周的培训, 主要培训一些与毕业设计内 容密切相关的专业知识, 包括理论知识方面与实践操作方 面。由于这些学生前三年的知识掌握得很不扎实, 所以在 理论知识方面就需要有针对性地给学生重复讲解一些做毕 业设计时需要运用到的重要理论知识。而对于工科专业的 学生来说, 实践动手能力更是至关重要的, 所以在首次的 集中培训期间, 重点是放在动手操作上, 教师首先设计好 一些案例, 然后让学生一步步跟着老师操作, 直至让每个 同学都真正搞懂、学会独立完成一个简单的系统为止。经 过这一阶段的培训, 能为学生开展毕业设计项目打下较好 的基础。

在接下来的阶段, 对这些学生采取集中管理与分别辅 导相结合的模式开展毕业设计。所谓集中管理就是对他们 进行分阶段集中培训, 每次培训后都布置相应的任务并要 求在规定时间内完成; 所谓分别辅导就是指导小组中的每 个教师负责具体指导自己所带的学生, 根据每个学生开展 的毕业设计项目分别指导, 并检查每个学生每一阶段该完 成的任务, 采取责任到位。后期集中培训大致这样安排: 总共安排 6 次左右的集中讲解, 主要内容包括介绍如何查 阅文献及进行外文翻译 (要求在规定时间内上交查阅的相 关文献目录及译文); 如何写文献综述 (要求在规定时间内 上交文献综述报告); 如何写开题报告 (要求在规定时间内 上交开题报告); 开发毕业设计项目的大致实现方法与步骤 (规定每一阶段完成的时间); 如何撰写毕业论文 (规定上 交初稿的时间); 根据已上交的初稿中出现的问题介绍如何 修改毕业论文 (规定终稿完成的时间); 如何准备毕业论文 答辩 (上交答辩用 PPT) 等。经过把毕业设计这一较庞大 的任务分解成每一阶段的子任务, 并指导督促学生在每个 阶段都认真按时完成, 毕业设计辅导班的学生最后大多能 顺利完成毕业设计项目并通过论文答辩。

\section{3 毕业设计内容的设计}

选择怎样的毕业设计项目让学生去完成是非常关键 的, 难度太大会打击学生的积极性, 而且有可能最后完不 成任务; 太简单的话达不到毕业设计的目的与要求。所以, 毕业设计管理工作小组的教师们根据学习困难生的实际水 平与能力, 花了大量的精力去收集各类难易程度适当的毕 业设计题目, 把这些课题集中起来, 建立了毕业设计题目 库。每一届的学生都让他们自己从中挑选感兴趣的课题, 同时教师们对题库里的题目也不断地进行更新与修改, 使 得尽量跟上时代的步伐, 使课题具有一定的新颖性与实用 
性。

\section{3. 产生的效果}

通过将学习困难生组织起来, 统一集中管理, 分阶段 进行培训, 因材施教, 根据学生的能力情况作特殊对待, 有效地解决了学习困难生往往在最后一年浑浑哥哥地度 过、无法较好地完成毕业设计任务的这个难题, 同时, 更 重要的是使学生在顺利完成毕业实习与毕业设计这个教学 环节时, 让他们能真正地增长一些专业知识与专业本领, 提高独立运用所学知识解决实际问题的能力, 增强综合运 用能力与实践操作能力, 最后能顺利毕业、顺利就业。

经过几年的摸索, 几届自愿报名参加学习困难生辅导 班的学生均已顺利完成最后一年的文献阅读与毕业设计环 节, 完成的毕业论文质量与毕业设计作品质量都较以往整 体有了较大的提高, 且在毕业时也都能顺利找到就业单位。 我们的学习困难生的毕业设计指导工作已逐步走向规范化 与正规化。

\section{4. 结束语}

我们在计算机专业开展的学习困难生毕业设计指导方 式改革的实践, 已经取得了较好的效果。通过改革, 使每个 参加的学习困难生都增长了专业知识, 提高了专业本领, 并增强了综合应用能力。这套方法将逐步推广到其它专业, 使得更多的学习困难生收益, 为他们最终顺利完成四年学 业画上完美的句号。

\section{参考文献(References)}

[1] F. Lv, "Exploration and practice of undergraduate engineering course's graduation project reform," Journal of Changchun University of Science and Technology, vol. 5, no. 1, pp. 156-157, January 2010.

[2] Y.-F. Wang, et al, "Exploration on computer science bachelor's graduate design," Computer Education, no. 1, pp. 77-79, January 2010.

[3] Y. Gao, "Analysis of the norms of administration for graduation project and practical ability cultivation of college graduates," Journal of Xi'An University of Post and Telecommunications, vol. 14, no. 3, pp. 156-159, May 2009. 